\title{
Frequency Response of an Aircraft Wing with Discrete Source Damage Using Equivalent Plate Analysis
}

\author{
T. Krishnamurthy \\ NASA Langley Research Center, Hampton, VA 23681, U.S.A. \\ and \\ Lloyd B. Eldred ${ }^{\dagger}$ \\ Lockheed Martin Mission Services, Hampton, VA 23681, U.S.A.
}

\begin{abstract}
An equivalent plate procedure is developed to provide a computationally efficient means of matching the stiffness and frequencies of flight vehicle wing structures for prescribed loading conditions. Several new approaches are proposed and studied to match the stiffness and first five natural frequencies of the two reference models with and without damage. One approach divides the candidate reference plate into multiple zones in which stiffness and mass can be varied using a variety of materials including aluminum, graphite-epoxy, and foam-core graphite-epoxy sandwiches. Another approach places point masses along the edge of the stiffness-matched plate to tune the natural frequencies. Both approaches are successful at matching the stiffness and natural frequencies of the reference plates and provide useful insight into determination of crucial features in equivalent plate models of aircraft wing structures.
\end{abstract}

\section{Introduction}

Understanding the effects of discrete source damage (e.g. uncontained rotor burst) on the response of aircraft structures is necessary to improve the survivability of future aircraft to adverse damage events. Rapid modeling and analysis methods are also key requirements to generate static and dynamic load constraints for an adaptive control system and for vehicle health management in the presence of structural damage. In order to generate damagedependent structural constraints for an adaptive control system and to verify that the control frequency is sufficiently separate from the structural frequencies, it is necessary to determine the frequency response of the structures in the presence of damage. Frequency estimation is also necessary to determine the flutter boundary changes due to the damage. Hence, there is a need to develop methods to determine the frequency response of the aircraft wing in the presence of damage. One such method is equivalent plate analysis.

Equivalent plate analysis is often used to replace the computationally expensive detailed finite element analysis in initial design stages or in conceptual design of aircraft wing structures [1]. In equivalent plate modeling, the model characteristics are represented with polynomials, which require only a small fraction of the input data that would be required by a corresponding detailed finite element model. The equivalent plate model can also be used to design wind tunnel models [2]. For example, the stiffness characteristics can be matched for the wing box of a full-scale flight vehicle model while satisfying strength-based requirements. An equivalent plate analysis procedure based on the Ritz method was proposed at NASA Langley Research Center as early as 1986 [3]. In the Ritz method-based equivalent plate theory, the aircraft wing structure is modeled with several trapezoidal segments. Several modifications and improvements to the initial theory proposed resulted in the development of a structural analysis code ELAPS (Equivalent Laminated Plate Solution) [4,5]. The ELAPS code solutions predict the displacement, stress, and mode shape responses within five to ten percent of a comparable finite element solution [6]. However, the Ritz method-based equivalent plate theory in the ELAPS code is not easily amenable to implementation in general-purpose commercial structural finite element analysis codes.

\footnotetext{
* Aerospace Engineer, Durability, Damage Tolerance and Reliability Branch, Associate Fellow, AIAA

${ }^{\dagger}$ Senior Engineer, Durability, Damage Tolerance and Reliability Branch, Senior Member, AIAA
} 
Recently, an equivalent plate procedure was developed to provide a computationally efficient means of matching the stiffness and frequencies of flight vehicle wing structures for prescribed loading conditions [7]. Equivalent plates were generated to match the stiffness of stiffened-panels without damage and then with damage. For both stiffened panels, the equivalent plate models accurately reproduced the deformation of a corresponding detailed model for the given loading conditions. Once the stiffness was matched, the equivalent plate models were then used to predict the frequencies of the panels. Two analytical procedures using a lumped-mass matrix were used to match the first five frequencies of the corresponding detailed model. In both procedures, the lumped-mass matrix for the equivalent plate was constructed by multiplying the diagonal terms of the consistent-mass matrix by a proportionality constant. In the first procedure, the proportionality constant was selected such that the total mass of the equivalent plate was equal to that of the detailed model. In the second method, the proportionality constant was selected to minimize the sum of the squares of the errors in a set of pre-selected frequencies between the equivalent plate model and the detailed model. The equivalent plate models reproduced the fundamental first frequency accurately in both the methods. It was observed that changing only one mass scaling parameter in the equivalent plate model did not provide enough flexibility to match all of the frequencies. Hence, there is a need to develop a better equivalent plate model to predict the natural frequencies accurately. In this paper, the following two approaches will be examined to match the frequency response of the wing structures using an equivalent plate model.

1. The equivalent plate is generated by matching both the stiffness and the frequencies of the flight vehicle wing structure simultaneously.

2. The equivalent plate is generated by only matching the stiffness of the flight vehicle wing structure, as discussed in the previous work. The frequency response of the equivalent plate is matched using distributed point masses while holding the stiffness fixed.

Several candidates for the equivalent plate are presented and compared, including composite layups, foam core sandwiches, a multi-zone aluminum plate, and an aluminum plate with discrete point masses along its edges. These approaches are intended to produce a model suitable for numerical analysis of the structure. In developing these theoretical models discussed in the present work, no a priori requirements are placed on the suitability for manufacturing. However, this issue is examined in the discussion at the end of the paper.

The paper is constructed as follows. First, an optimization process that generates an equivalent plate model will be discussed. Results from the previous work [7] where only stiffnesses were successfully matched will be recalled. Next, three different candidate equivalent plate models created by simultaneous stiffness and frequency matching will be examined. Then, results from the point mass distribution method of matching natural frequencies will be presented. The fundamental geometric differences between the reference and equivalent plate models make exactly matching natural mode shapes unrealistic. Yet, it is of interest to verify that this global behavior corresponds in a general sense. A comparison will be made between the mode shapes of the reference models to some of the equivalent plate models. Finally, merits of the various candidate approaches will be discussed.

\section{Equivalent Plate Theory Development}

A brief review of the procedure described in the previous work [7] is in order, as the same process is used in the current work to develop the present equivalent plate analyses. Various candidate equivalent plate approaches are examined to approximate the response of a more complex wing structure. The candidate equivalent plates are considered to match the stiffness of the reference structure when they exactly match the deformation of the reference for a prescribed set of loading and boundary conditions. Frequency (vibrational) response is considered to match when the natural frequencies are equal. Mode shapes will be examined in this paper, but are not part of the numerical process to generate equivalent plates. The procedure to create the equivalent plate model is described as follows:

1. Obtain the displacement field of the flight vehicle by performing full scale finite element linear static analysis for the given loading and boundary conditions.

2. Fix the dimensions of the equivalent plate planform.

3. Construct the finite element model of the equivalent plate.

4. If the nodal locations of the equivalent plate model are different from that of the full-scale fight vehicle finite element nodes, interpolate displacements at the equivalent plate nodal locations. In the present study, 
the interpolated displacements at the equivalent plate nodal locations are denoted as the flight vehicle reference displacements.

5. Find the displacement distribution of the equivalent plate by performing linear static analysis for a given thickness distribution of the plate and determine the optimum thickness distribution of the equivalent plate by minimizing the sum of the differences between the flight vehicle reference displacements and the equivalent plate model displacements at all nodal locations.

The general purpose finite element code ABAQUS ${ }^{\circledR}[8]^{\ddagger}$ is used for the finite element analysis. The optimization code DOT ${ }^{\circledR}[9]$ is used in step 5 to minimize the difference in displacements between the reference and equivalent models. The objective function $\Phi$ for the optimization problem can be stated as

$$
\Phi=\sum_{i=1}^{N}\left(w_{i}^{E}-w_{i}^{F}\right)^{2}
$$

where,

$N$ is the number of finite element nodes in the equivalent plate model;

$w_{i}^{E}$ is the displacement at the $i^{\text {th }}$ node of the equivalent plate; and

$w_{i}^{F}$ is the interpolated displacement of the flight vehicle at the location

of the $i^{\text {th }}$ node of the equivalent plate.

The objective function $\Phi$ in Equation (1) is minimized in the optimization to obtain the thickness distribution of the equivalent plate. A similar function representing the error in frequency response is used as a constraint function

$$
g=\sum_{i=1}^{M}\left(\frac{\left(\omega_{i}^{E}-\omega_{i}^{F}\right)}{\omega_{i}^{F}}\right)^{2}
$$

where,

$M$ is the number of natural frequencies to be matched

$$
\begin{aligned}
& \omega_{i}^{E} \text { is the } i^{\text {th }} \text { natural frequency of the equivalent plate; and } \\
& \omega_{i}^{F} \text { is the } i^{\text {th }} \text { natural frequency of the flight vehicle }
\end{aligned}
$$

\section{Reference Models}

The equivalent plate procedure is demonstrated on a set of simple structures that simulate a cantilevered wing. In the first model, a stiffened panel with two blade stiffeners without damage is considered. In the second model,

* Trade names and trademarks are used in this report for identification only. Their usage does not constitute an official endorsement, either expressed or implied, by the National Aeronautics and Space Administration. 
the stiffened panel is modified to include discrete source damage in the form of a circular hole in the center of the panel.

\section{Model 1: Stiffened-Panel without Damage:}

The two-blade stiffened panel used to generate the equivalent plate model is shown in Figure 1. The dimensions, material properties and the loading used in the analysis are also shown in the figure.

The plate is completely fixed (cantilevered) at the edge CD. Two types of loading are applied simultaneously at the free edge $\mathrm{AB}$. The first is a uniform vertical displacement $-w_{B}$ that is applied at the free edge $\mathrm{AB}$ to simulate a bending loading. The second is a torsional loading that is simulated by linearly varying the vertical displacement from $+w_{T}$ at point $\mathrm{A}$ to $-w_{T}$ at point $\mathrm{B}$.

The finite element model of the stiffened panel and its equivalent plate model are shown in Figures 2 and 3, respectively. The nodes in the equivalent plate exactly match the nodes in the stiffened panel, thus eliminating the need for displacement interpolation in step 4 of the optimization procedure. Since the bending and torsional loads are simulated by applying displacement boundary conditions at the free edge, the total load applied is obtained by summing the reactions at the fixed edge in each of the models.

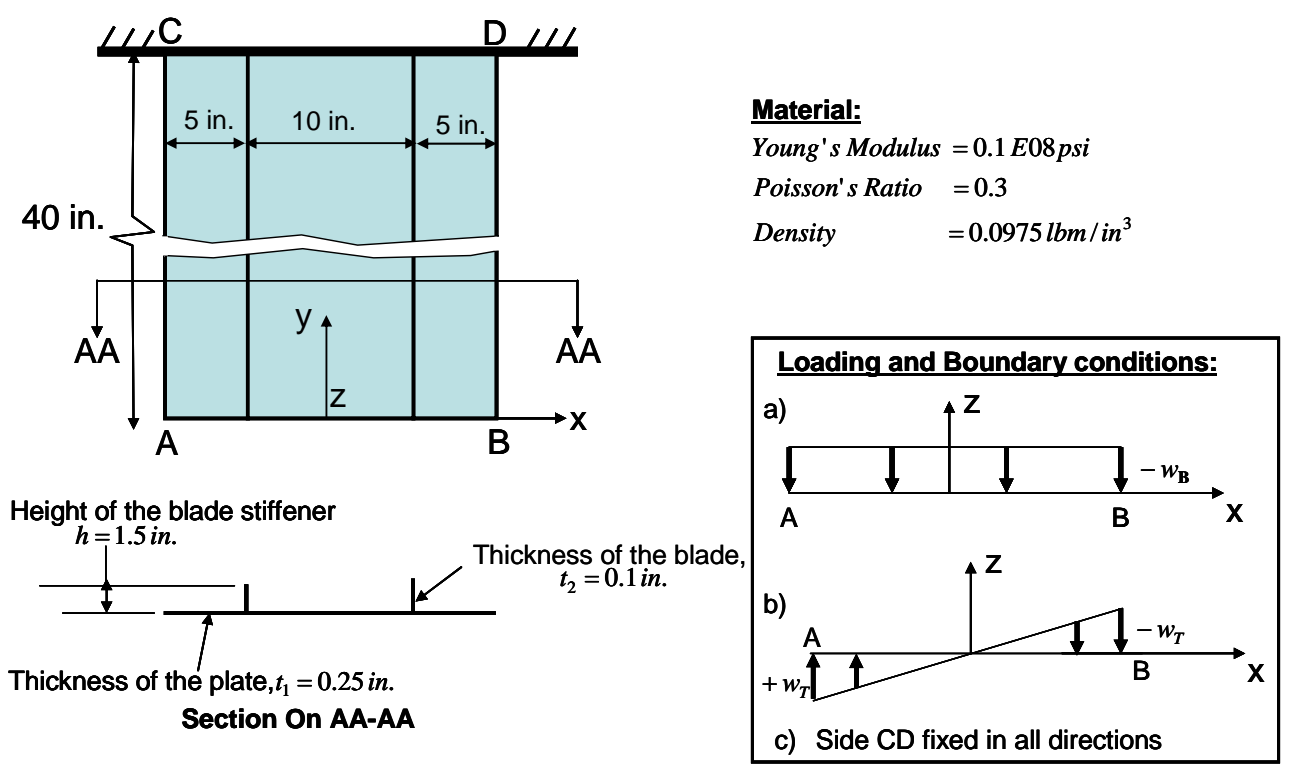

Figure 1. Geometry of the stiffened panel used in the equivalent plate generation

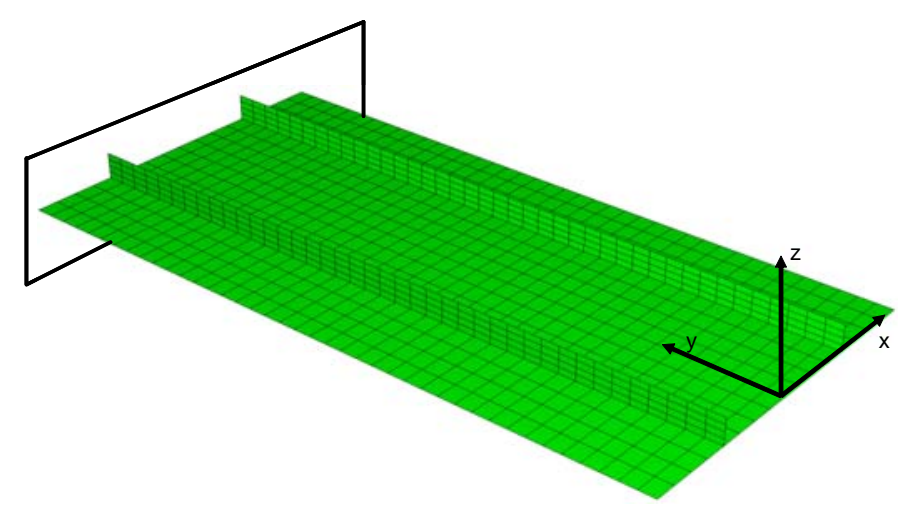

Figure 2. Finite element model of the stiffened panel reference model 


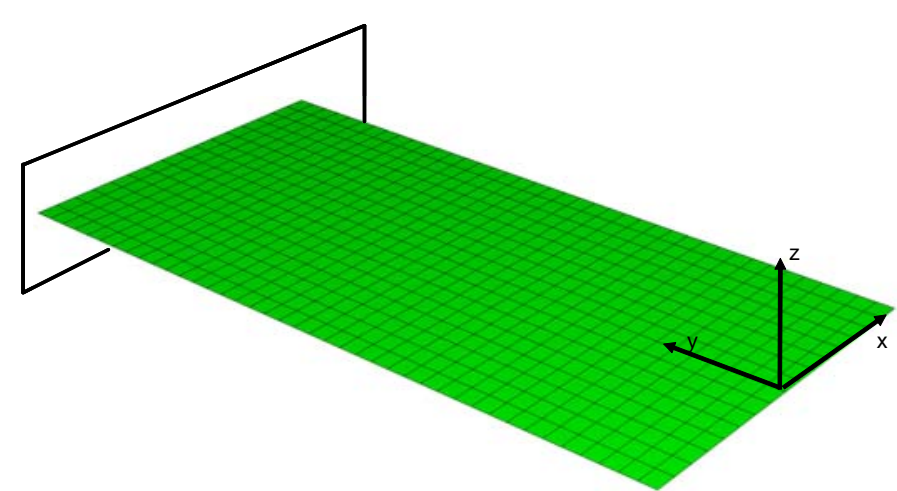

Figure 3. Finite element model of the equivalent plate

\section{Model 2: Stiffened-Panel with Circular Damage:}

In this model, the stiffened panel shown in Figure 1 is modified by introducing damage in the form of a 2.5 inch circular cut-out at the center. The dimensions, properties and loading, as shown in figure 4 . The stiffeners are cut at locations in the middle as shown schematically the figure. The damage size in the stiffened-panel and the equivalent plate are kept the same. Figures 5 and 6 illustrate the finite element models for the reference case and for the equivalent plate.
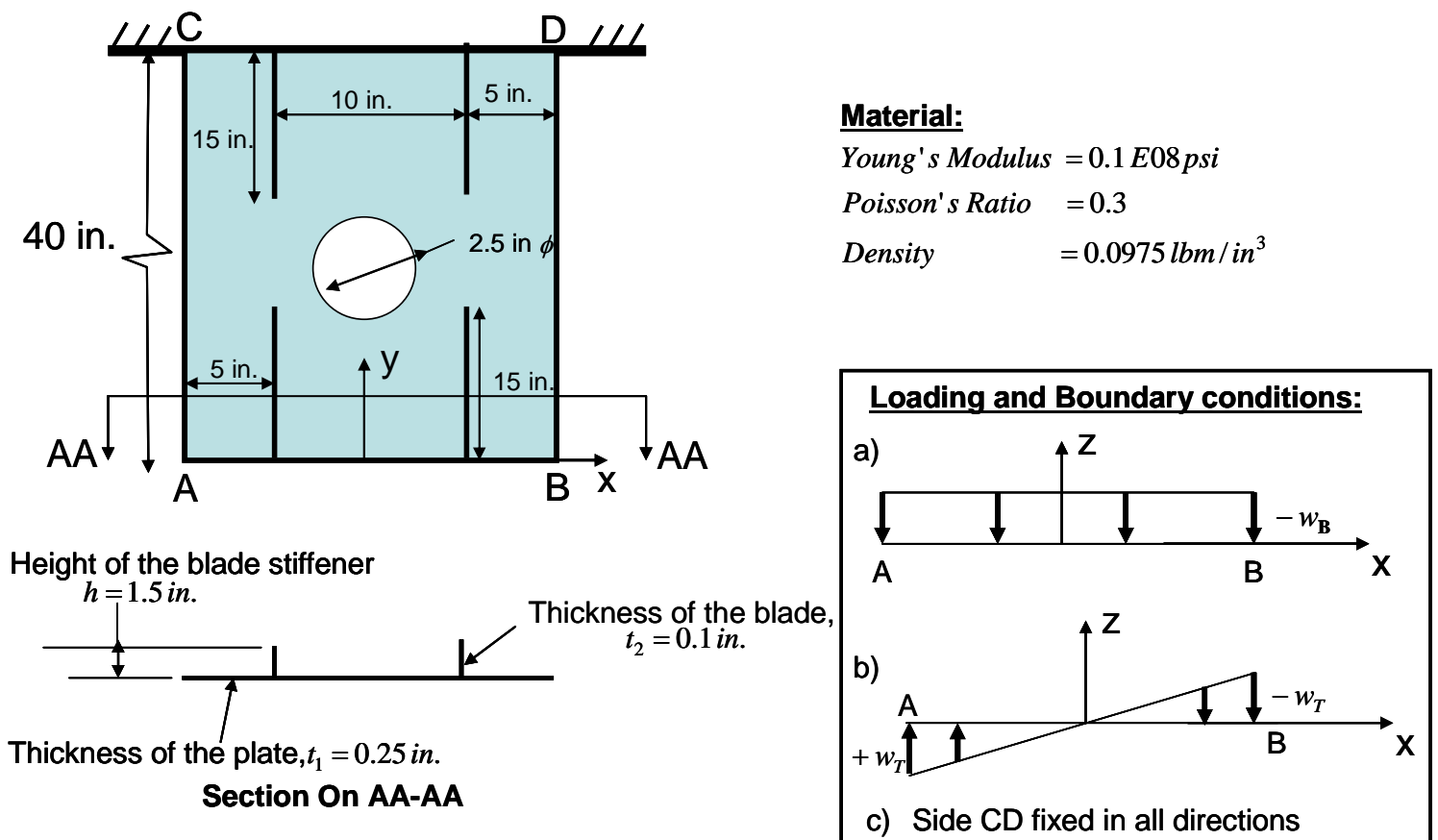

Figure 4. Geometry of the stiffened panel with circular damage used in the equivalent plate generation 


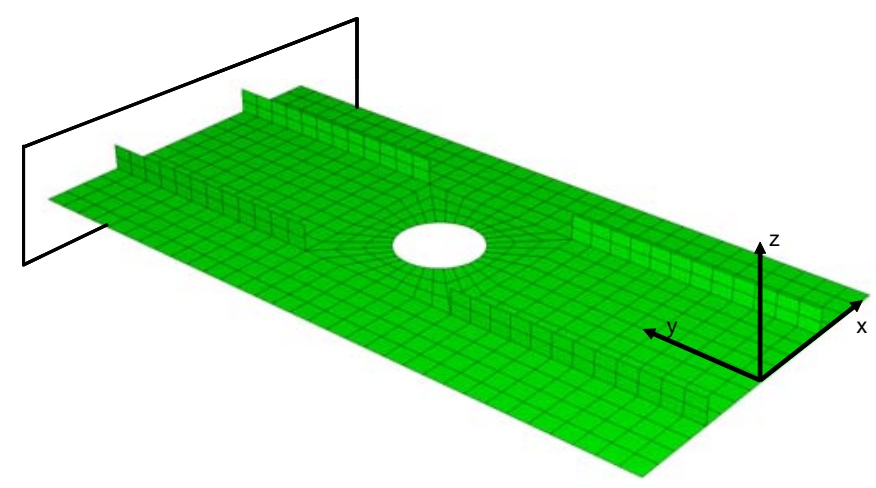

Figure 5. Finite element model of the reference stiffened panel with circular damage

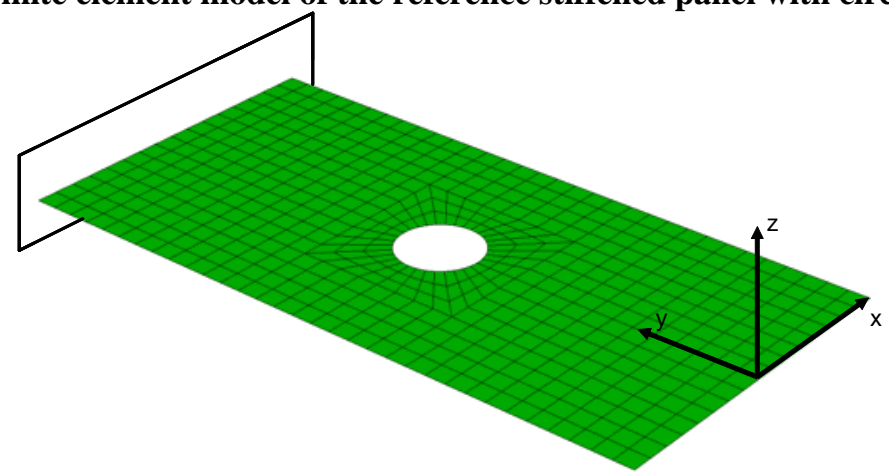

Figure 6. Finite element model of the equivalent plate with circular damage

\section{Matching Stiffness with an Equivalent Plate}

In the previous paper [7] the equivalent plates accurately matched the static deflection response of both reference models. In other words, the stiffness of the reference plates and the equivalent plates are the same if the deformations are the same for the given set of imposed loads and boundary conditions. Figure 7 compares the static response of both the reference and equivalent plates for the test models with and without damage. The imposed torsion is difficult to discern in the deflected shape, but can be noted in the colored contours indicating total deflection magnitude.
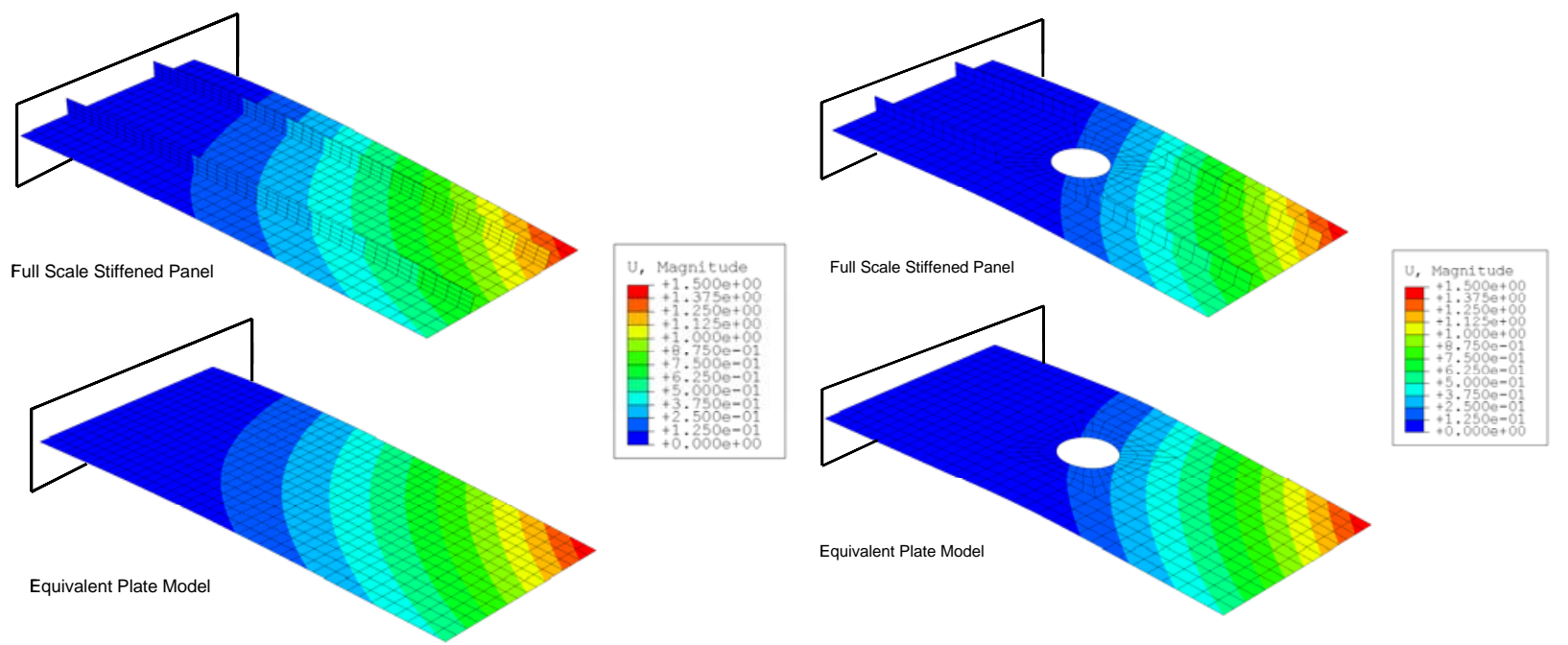

Figure 7. Comparison of static response for both damaged and undamaged plates. 
All of the equivalent plate approaches discussed in the remainder of this paper successfully matched the deformation of the reference plates. Hence, the deformation comparison of the equivalent plate with the reference solution is not repeated for other cases studied in this paper.

\section{Matching Frequencies and Stiffness Simultaneously}

The previous work [7] was not successful at matching the natural frequencies after matching the reference stiffness. Therefore, a new approach where both frequency and stiffness are matched simultaneously was developed. A variety of material choices and model configurations were examined. These are a multiple zone aluminum plate, a five-ply composite plate, and a multiple zone foam core composite sandwich. In developing these models, no requirement is placed on the suitability for manufacturing of the resulting equivalent plates.

\section{A. Zonal Metallic Approach}

In the interest of improving the accuracy of the natural frequency match between the equivalent plate candidate and the reference model, a new approach was designed for the equivalent plate. The plates are divided into rectangular zones with finite elements assigned to zones based on the location of their centroid. Each zone was assigned two design variables corresponding to thickness and density which were assumed constant across the zone. These design variables were optimized with the stiffness matching equation (1) as the objective and the frequency matching equation (2) as the constraint.

Various numbers of zones were considered, including a different number in the two directions. As a first step, a stiffness-only equivalent plate was generated by omitting the frequency matching constraint. Only one zone was needed to satisfy the stiffness objective. More zones were required to satisfy both conditions.

Dividing the plate into sixteen zones, with four equal length zones in the chordwise and in the spanwise directions, was necessary to allow sufficient spatial variation of the mass to match the first five natural frequencies. Figure 8 shows the optimized values of the thickness and density design variables necessary to match both stiffness and the first five natural frequencies. The heavier and thicker zones tend to be on the leading and trailing edges of the plate, with the lighter and thinner zones occurring more often down the centerline. Figure 9 compares the natural frequencies of the reference and equivalent undamaged plates for the configuration shown in figure 1 . The agreement is excellent with all errors less than $0.3 \%$.

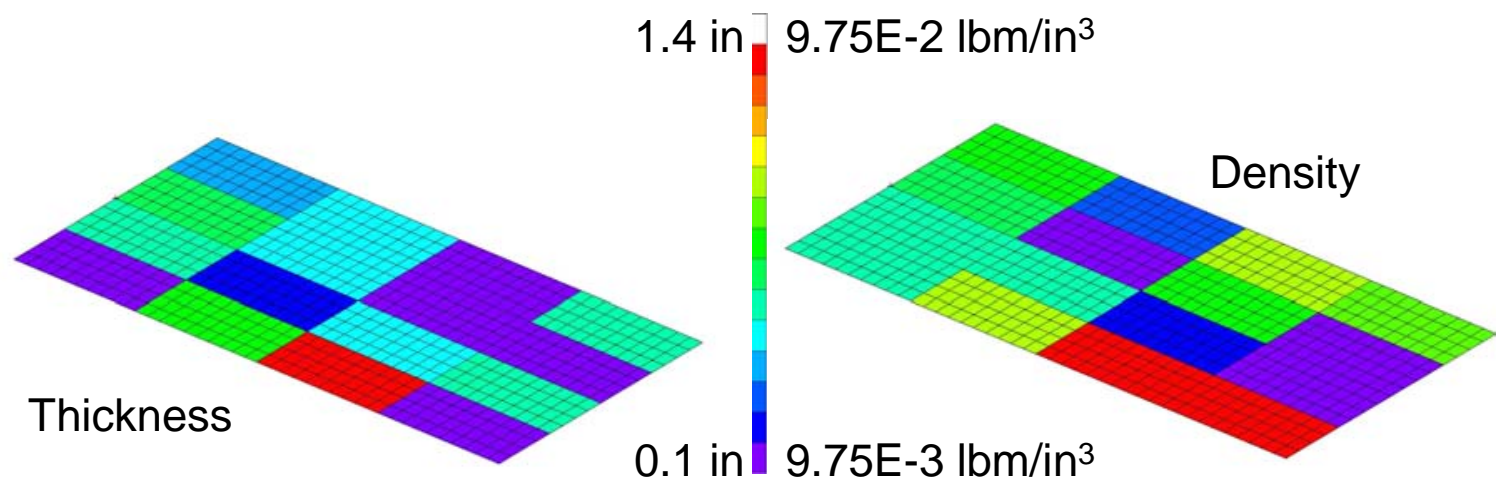

Figure 8. Undamaged Equivalent Plate Thickness and Density Distributions 


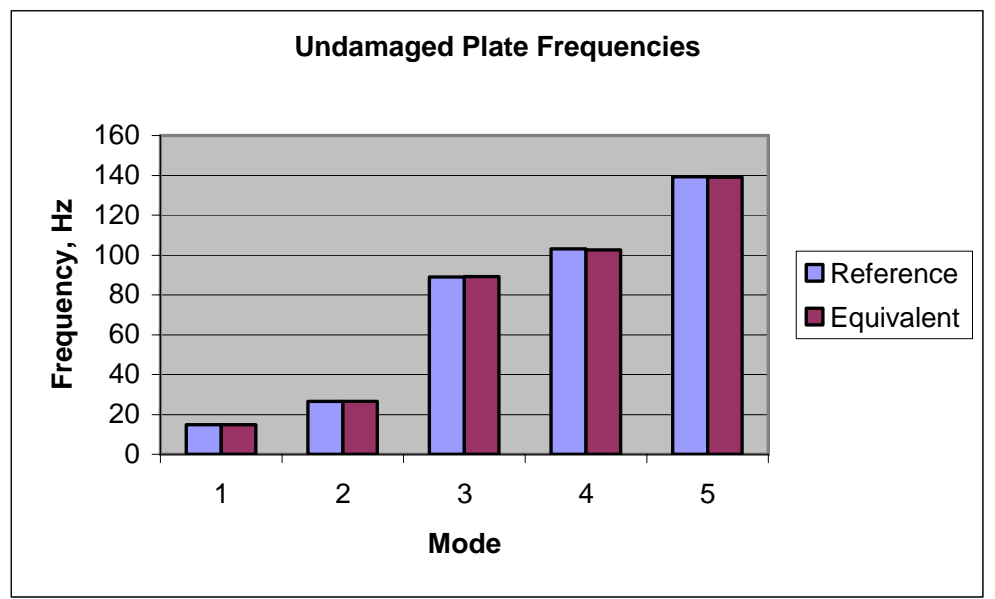

Figure 9. Comparison of natural frequencies of a 16 Zone Aluminum Plate to Reference Undamaged Model

Figure 10 shows the thickness and density results for a twenty-five zone equivalent plate configuration of the damaged reference model. The additional zones were necessary to produce frequency and stiffness results of similar quality to those of the undamaged model. Figure 11 compares the natural frequencies with all errors at $1 \%$ or less.

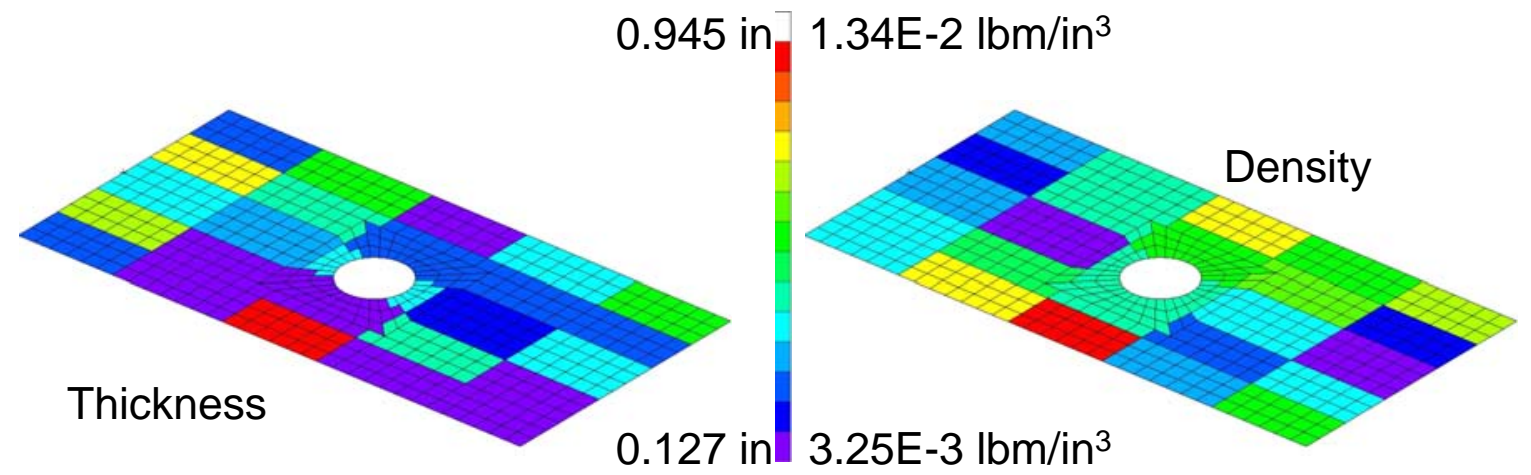

Figure 10. Damaged Equivalent Plate Thickness and Density Distributions

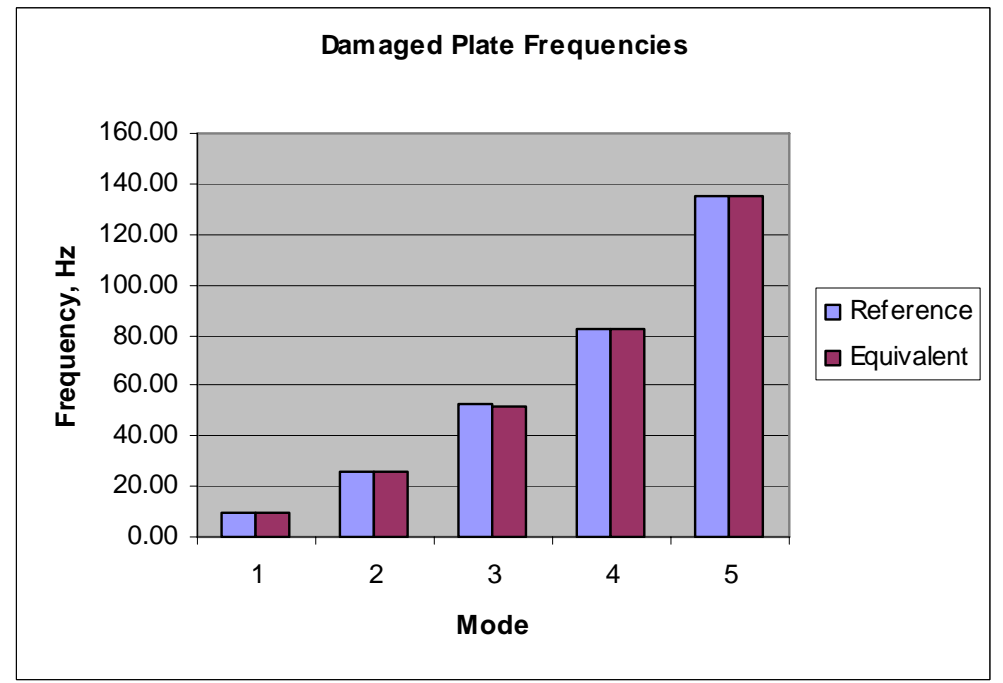

Figure 11. Comparison of natural frequencies of a 25 Zone Aluminum Plate to Reference Damaged Model

Note that the mass and thickness distributions are not attempting to mimic the positioning of the stiffeners in the reference plates. Rather, the distributions all show the similar result of positioning the thicker and denser plates 
(shown in red and yellow) at the outside edges of the equivalent plate, mimicking a more efficient stiffened plate. The regions corresponding to the zone between the reference stiffeners tend to have lower thicknesses and densities.

\section{B. Single Zone Composite Approach}

Two candidate equivalent plate numerical models were studied using composites. The first was a single zone, five-ply graphite epoxy plate where analyses were run varying each layer's thickness and orientation to satisfy the stiffness objective function (1) and the frequency matching constraint (2). Table 1 shows the material properties used in the analysis discussed here in section VI.B. and later in section VI.C. Table 2 lists the initial and final values for the thickness and orientation of each layer for the undamaged plate. A lower limit of 0.01 inches was chosen for numerical stability of the finite element analysis. The optimized result of a single 0.45 inch thick, 90 degree layup suggests that a single zone composite equivalent plate is not practical. Figure 12 compares the natural frequencies of the reference and equivalent plate. The maximum error shown is $14 \%$ for mode 1 .

Table 1: Composite and Foam In-Plane Properties

\begin{tabular}{|ccc|}
\hline Property & Gr/Ep & Foam \\
E1 & $1.85 \mathrm{E}+07$ & $1.00 \mathrm{E}+05$ \\
E2 & $1.54 \mathrm{E}+06$ & $1.00 \mathrm{E}+05$ \\
v12 & 0.28 & 0.3 \\
G12 & $8.50 \mathrm{E}+05$ & $3.85 \mathrm{E}+06$ \\
\hline
\end{tabular}

Table 2. Undamaged 5 Ply Composite Equivalent Plate Approach Result

\begin{tabular}{|c|cc|cc|}
\hline & \multicolumn{2}{|c|}{ Thickness, inches } & \multicolumn{2}{c|}{ Orientation, degrees } \\
Initial & Final \\
\hline 1 & 1.00 & 0.01 & 0 & 0 \\
2 & 1.00 & 0.01 & 45 & -90 \\
3 & 1.00 & 0.45 & 90 & 90 \\
4 & 1.00 & 0.01 & -45 & 90 \\
5 & 1.00 & 0.01 & 0 & 0 \\
\hline
\end{tabular}

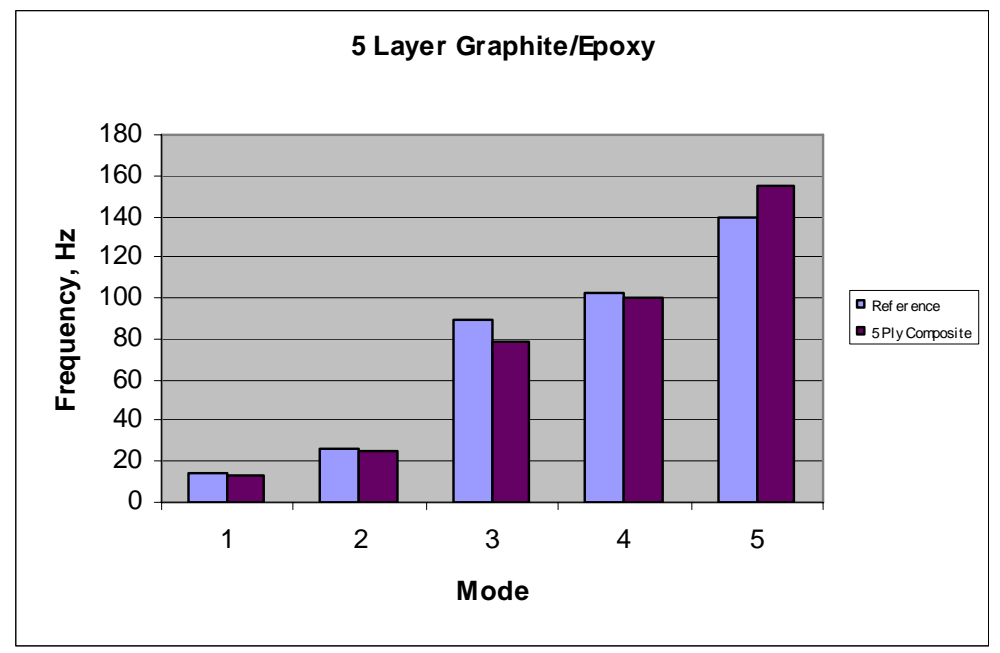

Figure 12. Undamaged Plate Frequency Match using a Composite Candidate

For the undamaged plate numerical model, the final equivalent plate's plies were all aligned and only one ply has a thickness above the minimum value. For the damaged plate, the results in Table 3 in general follow this trend. Figure 13 illustrates the frequency correspondence for the damaged plate. Here, the maximum error is $23 \%$ for mode 2. 
Table 3. Damaged 5 Ply Composite Equivalent Plate Approach Result

\begin{tabular}{|c|cc|cc|}
\hline & \multicolumn{2}{|c|}{ Thickness, inches } & \multicolumn{2}{c|}{ Orientation,degrees } \\
Layer & Initial & Final & Initial & Final \\
\hline 1 & 1.00 & 0.01 & 0 & 0 \\
2 & 1.00 & 0.28 & 45 & -90 \\
3 & 1.00 & 0.03 & 90 & 67 \\
4 & 1.00 & 0.02 & -45 & -61 \\
5 & 1.00 & 0.01 & 0 & 0 \\
\hline
\end{tabular}

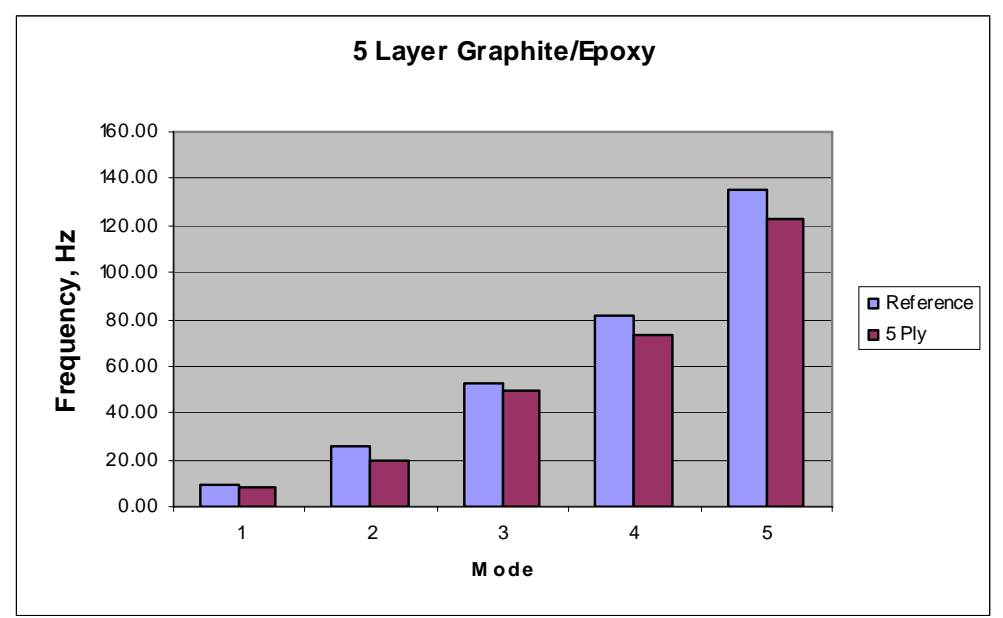

Figure 13. Damaged Plate Frequency Match using a Composite Approach

\section{Multi-zone Composite/Foam Sandwich Approach}

The second composite approach was a foam core sandwich with single ply graphite-epoxy face sheets, using the properties from table 1 . The composite face sheets used the thickness and orientation as design variables. The foam core had design variables of thickness and density. Sixteen zones were used for this approach.

Tables 4, 5 and 6 show the thickness of each layer in each of the sixteen zones. Table 4 illustrates the zone layout with the left column representing the fixed root of the plate and the right column the free edge. Table 7 shows the density result for the core in terms of the nominal foam density. The optimized results for the face sheet orientation were all zero degrees. The upper and lower face sheets produced identical thickness results with a thickness distribution across zones similar to the zonal aluminum result; Thicker panels were found at the edges of the plate. The foam core showed thicker results near the tip than at the root. Figure 14 shows the frequency matching success for the undamaged plate. The maximum error is 3\% for both modes 2 and 3.

Table 4: Undamaged Plate - Upper Face Sheet Thickness, inches

\begin{tabular}{|l|l|l|l|}
\multicolumn{4}{c}{ Leading Edge } \\
\hline 0.10 & 7.61 & 0.98 & 0.31 \\
\hline 0.10 & 0.16 & 1.01 & 0.13 \\
\hline 3.70 & 0.10 & 0.12 & 0.10 \\
\hline 3.11 & 0.76 & 1.64 & 4.13 \\
\hline
\end{tabular}

Trailing Edge

Table 5: Undamaged Plate - Foam Core Thickness, inches

\begin{tabular}{|l|l|l|l|}
\hline 0.14 & 0.34 & 2.37 & 2.36 \\
\hline 0.10 & 0.15 & 1.71 & 2.43 \\
\hline 0.70 & 0.10 & 0.14 & 0.10 \\
\hline 0.70 & 0.70 & 1.75 & 0.43 \\
\hline
\end{tabular}


Table 6: Undamaged Plate - Lower Face Sheet Thickness, inches

\begin{tabular}{|l|l|l|l|}
\hline 0.10 & 7.61 & 0.98 & 0.31 \\
\hline 0.10 & 0.16 & 1.01 & 0.13 \\
\hline 3.70 & 0.10 & 0.12 & 0.10 \\
\hline 3.11 & 0.76 & 1.64 & 4.13 \\
\hline
\end{tabular}

Table 7: Undamaged Plate - Core Density, scale relative to nominal foam

\begin{tabular}{|l|l|l|l|}
\hline 1.00 & 1.02 & 0.80 & 0.79 \\
\hline 1.00 & 0.95 & 0.81 & 0.74 \\
\hline 1.00 & 0.96 & 0.82 & 0.85 \\
\hline 1.00 & 1.02 & 0.82 & 0.90 \\
\hline
\end{tabular}

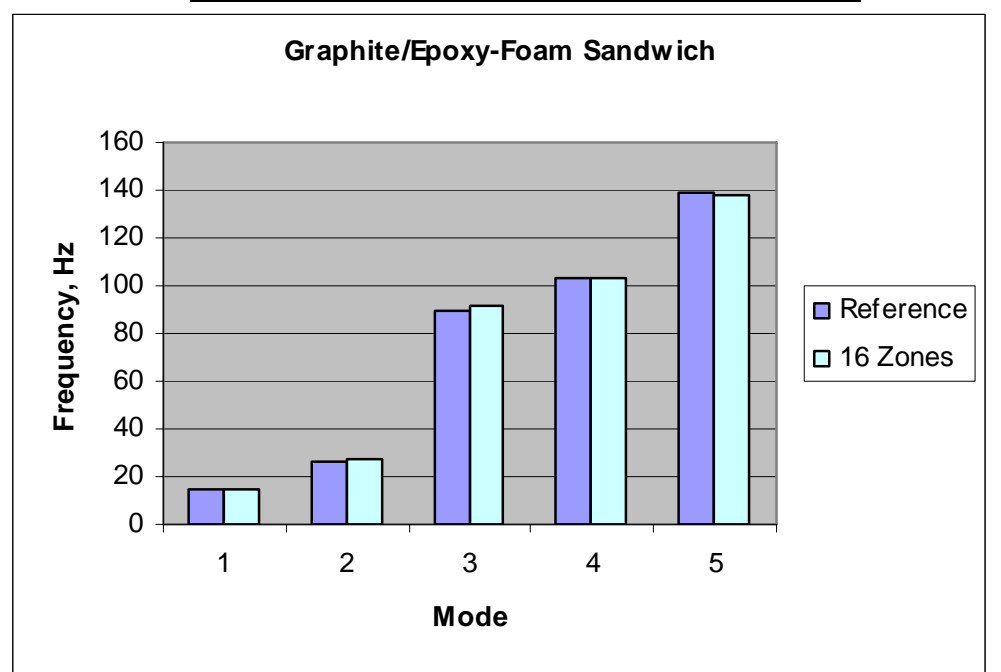

Figure 14. Undamaged Plate Frequency Match using a Foam Core Composite Sandwich Approach

Tables 8-11 contain the detailed results for the damaged plate as represented by the foam core sandwich approach using the layout indicated in Table 4. As with the undamaged plate, the optimized result for the composite sheet orientation were all zero degrees. The thickness distributions are significantly different, however. As before the upper and lower face sheets show identical thickness results, but with substantially lower values (all but four are at the optimization lower limit of 0.1 inch). The foam core shows significantly higher thickness variation than in the undamaged case. Figure 15 shows the frequency comparison for the damaged plate. The maximum error is $2 \%$ for mode 2 .

Table 8: Damaged Plate - Upper Face Sheet Thickness, inches

\begin{tabular}{|l|l|l|l|}
\hline 0.10 & 0.11 & 0.10 & 0.28 \\
\hline 0.10 & 0.10 & 0.10 & 0.10 \\
\hline 0.10 & 0.10 & 0.10 & 0.10 \\
\hline 0.10 & 0.19 & 0.24 & 0.10 \\
\hline
\end{tabular}

Table 9: Damaged Plate - Foam Core Thickness, inches

\begin{tabular}{|c|c|c|c|}
\hline 18.29 & 1.20 & 0.10 & 0.21 \\
\hline 0.40 & 0.10 & 0.10 & 0.21 \\
\hline 0.10 & 0.10 & 0.10 & 0.11 \\
\hline 0.10 & 0.35 & 0.10 & 0.10 \\
\hline
\end{tabular}

Table 10: Damaged Plate - Lower Face Sheet Thickness, inches

\begin{tabular}{|l|l|l|l|}
\hline 0.10 & 0.11 & 0.10 & 0.28 \\
\hline 0.10 & 0.10 & 0.10 & 0.10 \\
\hline 0.10 & 0.10 & 0.10 & 0.10 \\
\hline 0.10 & 0.19 & 0.24 & 0.10 \\
\hline
\end{tabular}


Table 11: Damaged Plate - Core Density, scale relative to nominal foam

\begin{tabular}{|l|l|l|l|}
\hline 0.01 & 0.05 & 0.05 & 1.17 \\
\hline 0.02 & 1.85 & 0.01 & 3.21 \\
\hline 0.01 & 1.30 & 0.16 & 0.09 \\
\hline 0.02 & 1.39 & 1.85 & 5.71 \\
\hline
\end{tabular}

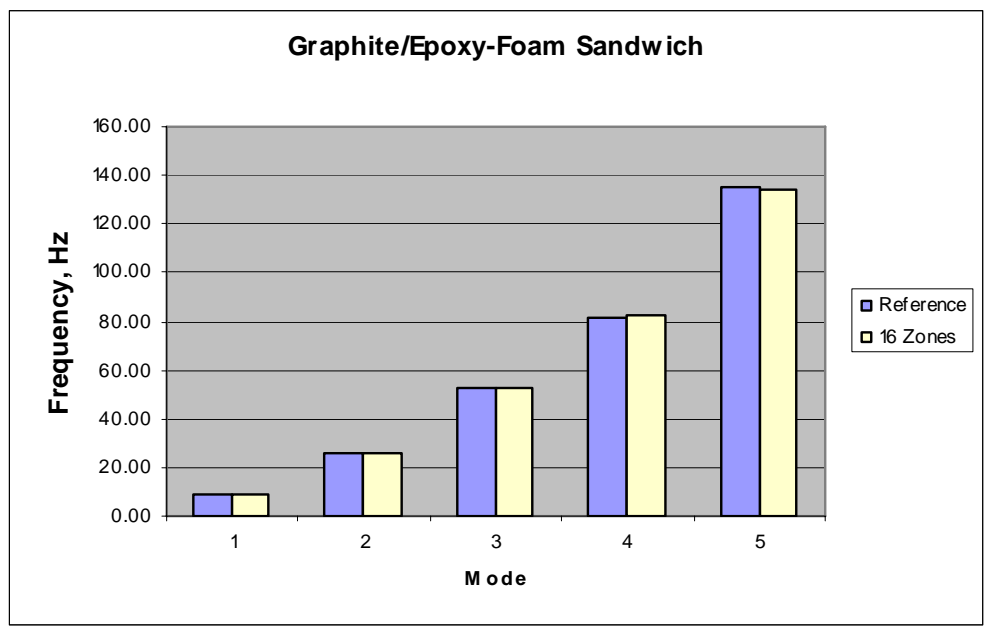

Figure 15. Damaged Plate Frequency Match using a Foam Core Composite Sandwich Approach

\section{Frequency Matching after Stiffness Matching}

The success of the zonal equivalent plates lead to a return to the previous paper's [7] polynomial variation in stiffness equivalent plate approaches. The earlier paper's thickness distributions that matched the reference plates' stiffnesses were retained. For this numerical model, nineteen to twenty-four point masses were distributed across the surface of the equivalent plates. The amount of mass added at each node was determined by a new optimization analysis designed to match the natural frequencies by using equation (2) as the objective rather than a constraint.

Another way to view this approach is that the equivalent plate's stiffness matrix is determined by optimizing the simple chordwise linear distribution of thickness that best matches the static response of the reference model. The equivalent plate's mass matrix completely replaces the aluminum plate's consistent mass matrix with a matrix representing lumped masses placed at selected nodes. These mass values are optimized to produce the best match to the reference plate's frequencies. Figure 16 compares the frequencies found in this approach to the reference frequencies. All errors were less than $0.3 \%$.

For the undamaged plate, twenty-four masses, distributed with three across the chord and eight spanwise, were required to produce the desired frequency accuracy. The lumped masses were in total 3.8 times the mass of the reference plate and were symmetric about the spanwise centerline. As with the zonal approach to mass distribution, the higher values were found near the fixed root and on the leading and trailing edges, with lower values down the centerline and at the tip. The values in Table 12 are percentages of the reference plate's mass. The specified coordinates are shown in Figure 1. The left column shows the weights at the nodes closest to the fixed root. The rightmost column contains the masses at the tip. Half of the mass variable results were approximately zero. These values were along the centerline and near the tip of the plate. 


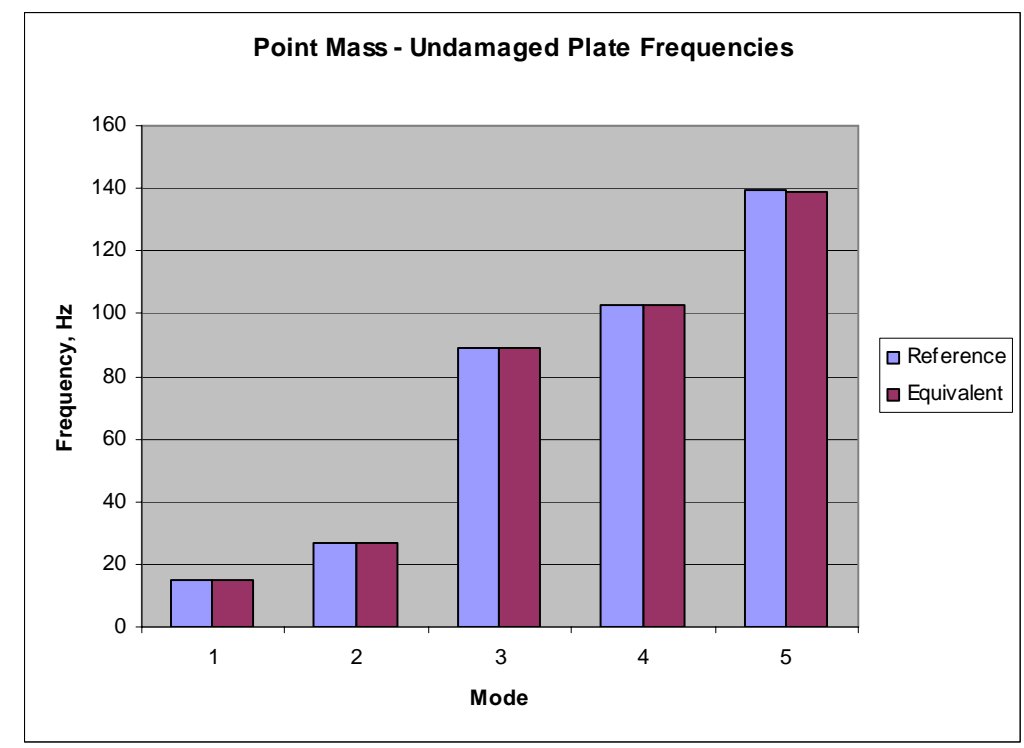

Figure 16. Undamaged Plate Frequency Match using a Point Mass Candidate

Table 12: Point Mass Distribution for the Undamaged Plate, see Figure 1 for coordinates

\begin{tabular}{|ccccccccc|}
\hline & $y=35$ & $y=30$ & $y=25$ & $y=20$ & $y=15$ & $y=10$ & $y=5$ & $y=0$ \\
$x=10$ & 0.7052 & 0.1462 & 0.0329 & 0.5341 & 0.2149 & 0.0000 & 0.0006 & 0.0005 \\
$\mathbf{x}=\mathbf{0}$ & 0.1197 & 0.4083 & 0.0043 & 0.0002 & 0.0013 & 0.0000 & 0.0000 & 0.0000 \\
$\mathbf{x}=-10$ & 0.7052 & 0.1462 & 0.0329 & 0.5341 & 0.2149 & 0.0000 & 0.0006 & 0.0005 \\
\hline
\end{tabular}

The damaged plate used nineteen point masses. They were distributed with five masses across the chord and four masses spanwise. No mass was assigned to the position within the damaged region. The total value of these point masses was approximately equal the reference plate mass when frequencies were matched. Figure 17 compares the equivalent plate and reference frequencies. All frequency errors were under $2 \%$.

The authors felt that this easier and more accurate vibrational equivalence, compared to the undamaged results, is due to the response of the plate being dominated by the damaged region. This region is significantly more flexible than the other regions due to the damage and the lack of stiffeners in the reference plate. A flat equivalent plate has a much easier task in matching the response of a region that is itself a flat plate. Table 13 shows the distribution of point masses. The result is non-symmetric, but does show the same trend of heavier values near the fixed root and on the leading and trailing edges.

Table 13. Point Mass Distribution for the Damaged Plate, see Figure 4 for coordinates

\begin{tabular}{|c|c|c|c|c|}
\hline & $y=30$ & $Y=20$ & $y=10$ & $y=0$ \\
\hline$x=10$ & 0.0946 & 0.2615 & 0.0257 & 0.0045 \\
\hline$x=5$ & 0.1213 & 0.0592 & 0.0712 & 0.0046 \\
\hline$x=0$ & 0.1037 & (hole) & 0.0227 & 0.0185 \\
\hline$x=-5$ & 0.0101 & 0.0117 & 0.0204 & 0.0687 \\
\hline$x=-10$ & 0.0084 & 0.0322 & 0.0272 & 0.0338 \\
\hline
\end{tabular}




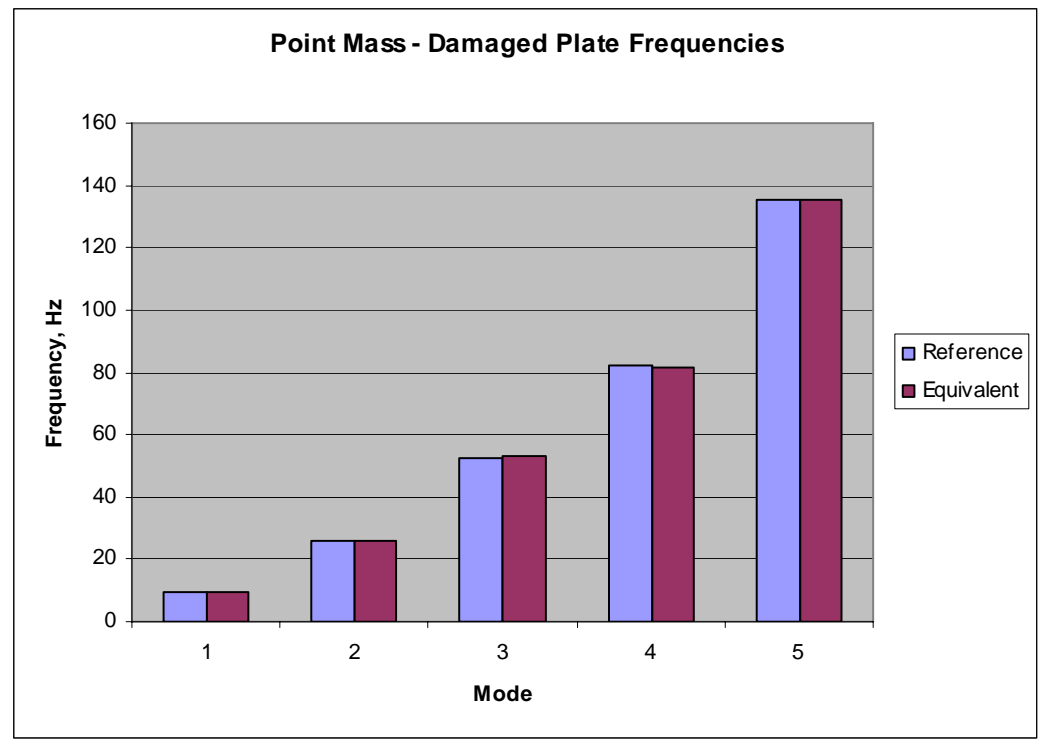

Figure 17. Damaged Plate Frequency Match using a Point Mass Approach

\section{Mode Shapes}

These equivalent plate models have done an excellent job of matching both the reference stiffness and frequencies. But, no attempt was made to control mode shape fidelity. Figures 18 and 19 show the mode shapes for the zonal aluminum candidate plates compared to the reference plates. Figures 20 and 21 compare the mode shapes produced by the point mass approach. The mode shapes for the other plates were examined, but are not presented here as they showed similar behavior to the modes shown.

The fundamental geometric differences between the reference and equivalent models make exactly matching natural mode shapes unrealistic. Yet, it is of interest to verify that this global behavior corresponds in a general sense. General correspondence means that modes that are bending-dominant or torsion-dominant should match. Producing corresponding mode shapes is more important for lower modes than for higher modes as these modes are the critical ones for flutter and divergence. The "curling" fifth mode of the reference structures is not well matched by the equivalent plates. 


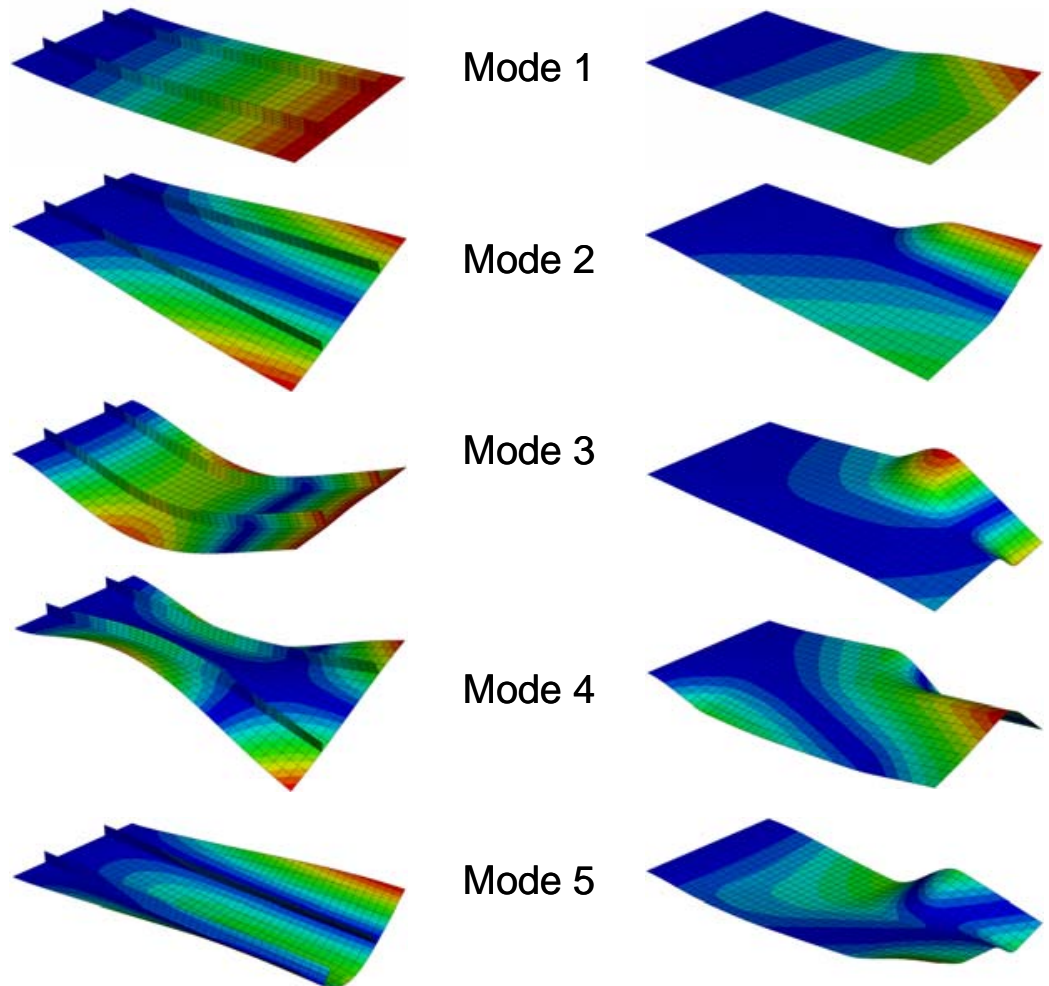

Figure 18. Undamaged Plate Mode Shape Match with Zonal Aluminum Approach

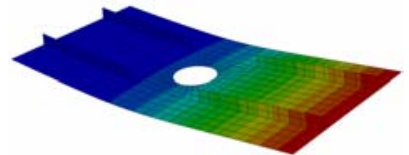

Mode 1
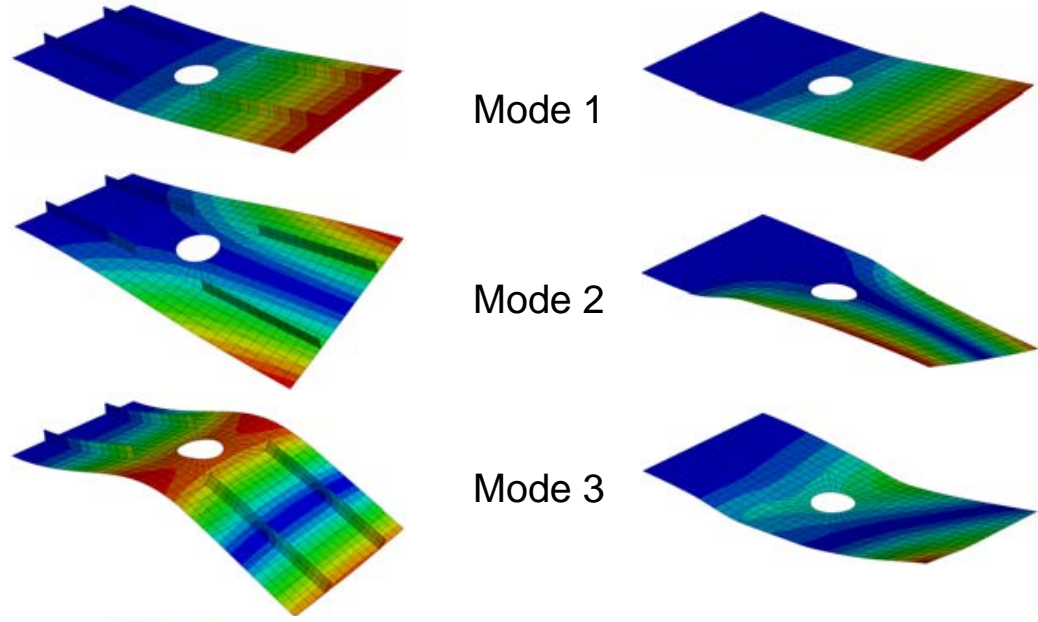

Mode 3
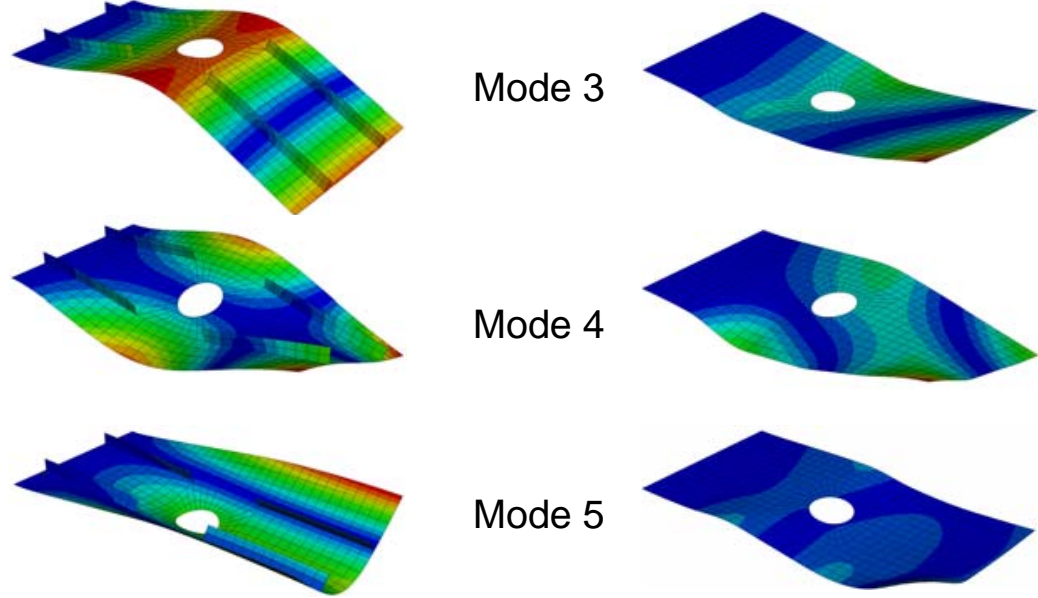

Mode 5

Figure 19. Damaged Plate Mode Shape Match with Zonal Aluminum Approach 


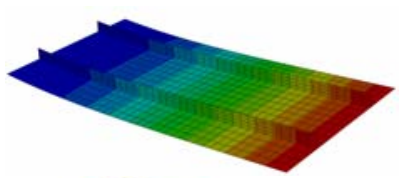

Mode 1

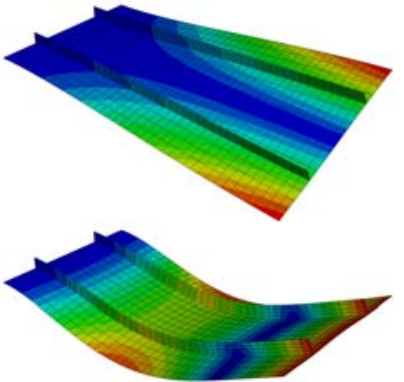

Mode 2

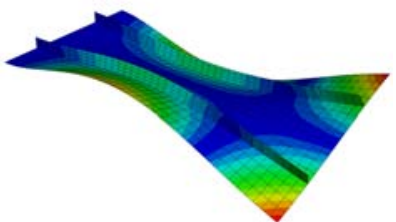

Mode 3
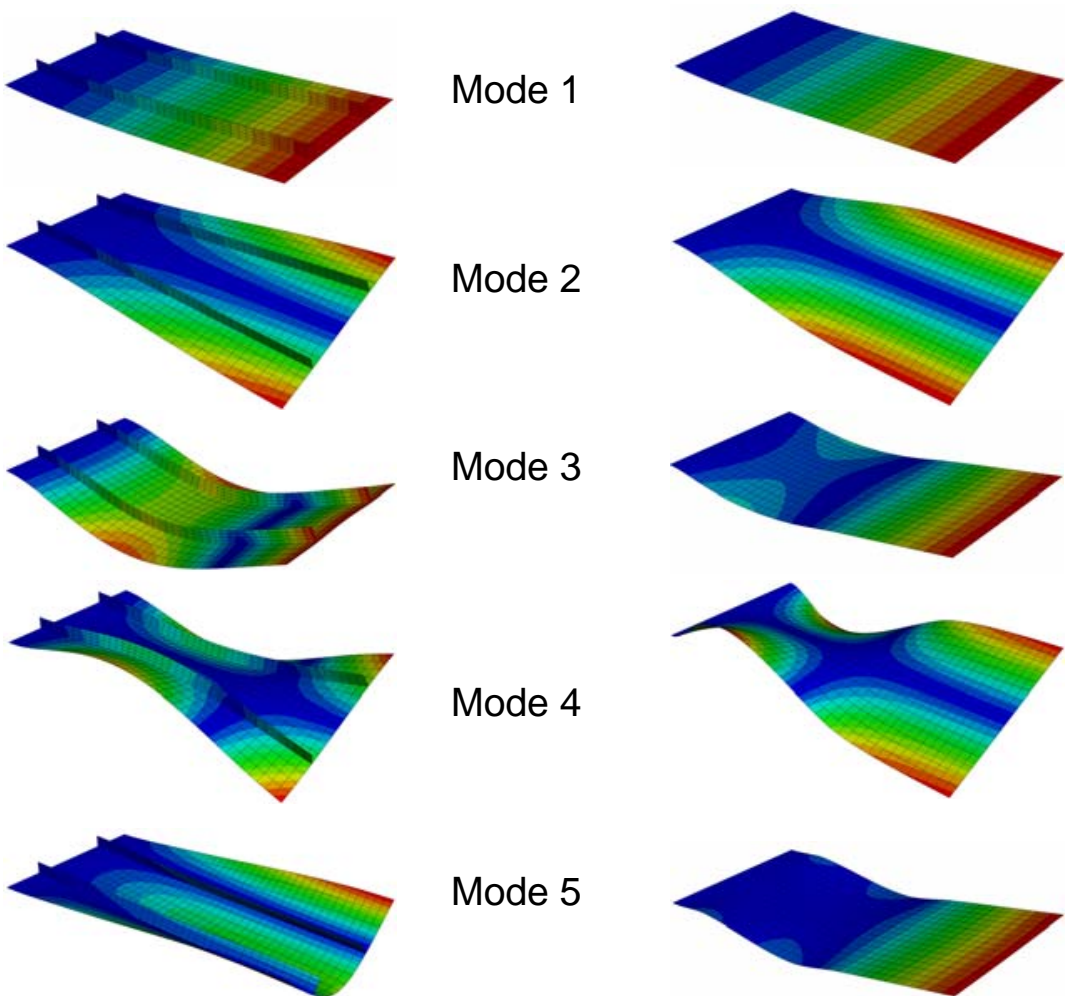

Mode 5

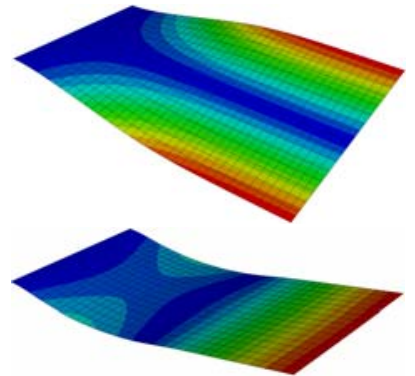

Mode 4

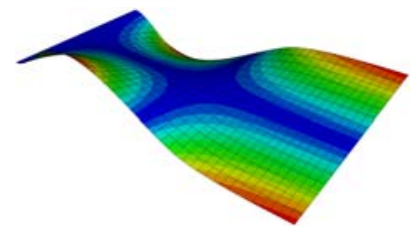

Figure 20. Undamaged Plate Mode Shape Match with Point Mass Approach

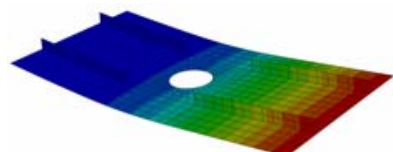

Mode 1
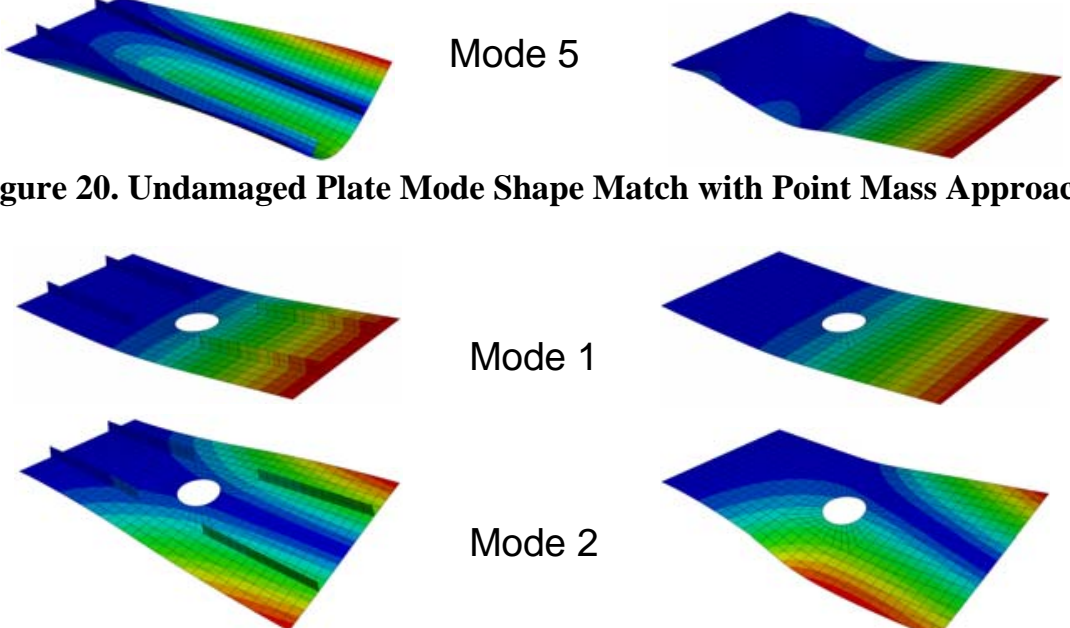

Mode 2

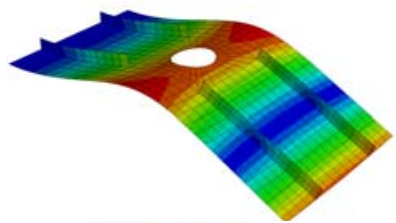

Mode 3
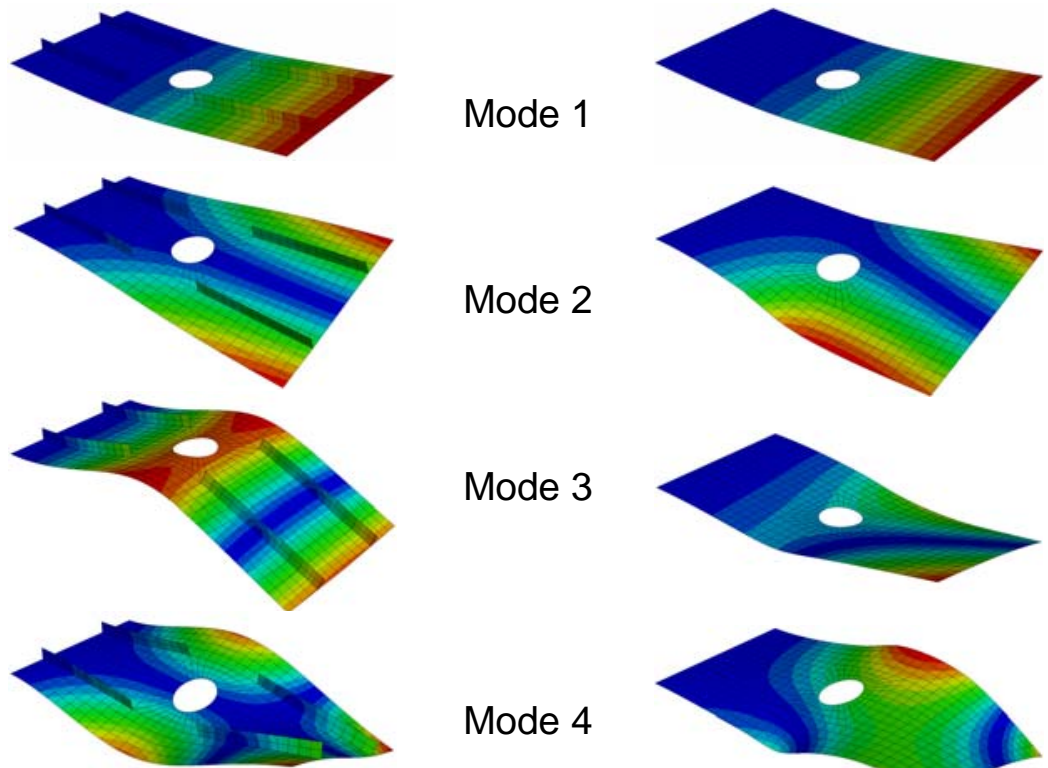

Mode 4
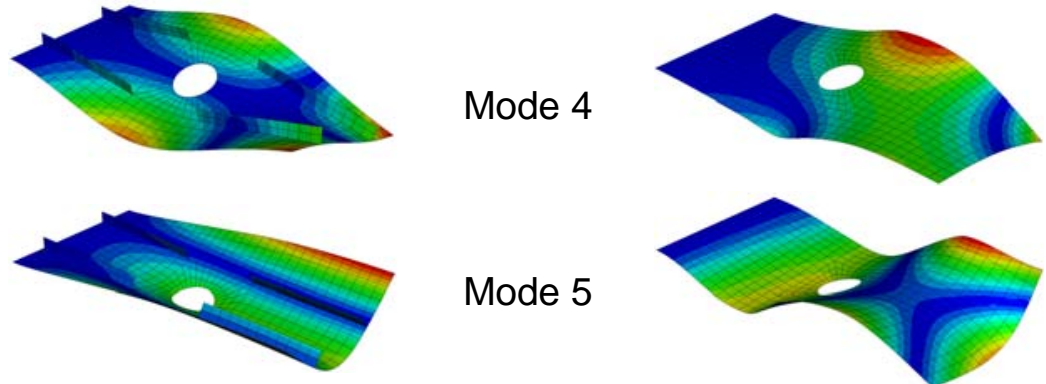

Figure 21. Damaged Plate Mode Shape Match with Point Mass Approach 


\section{Discussion}

One of the goals of this work is to develop an equivalent plate approach that could be constructed and tested in the laboratory and a wind tunnel. As a first step, each of the proposed approaches was used to create an equivalent plate model that satisfied only the stiffness criteria. These stiffness-only models were successfully generated in a single zone and were very suitable for construction as a test article. When the frequency matching constraint is added to the analysis, more zones are required and the resultant models are not generally well suited for manufacturing. At present, it appears that the equivalent plate can be fabricated and used in the simulation studies only satisfying the stiffness characteristics of the reference model. Physical models with many of the same characteristics as the point mass approach and the variable thickness sandwich plate could be possible if appropriate manufacturing constraints were added to the analysis.

Equivalent plates are inherently structurally inefficient compared to the stiffened reference plates, and are thus heavier. Therefore, matching the reference models' frequency responses is problematical. With the exception of the single zone composite plate, the approaches described in this paper do, in large degree, manage to succeed at matching frequencies. But, their results are only useful for numerical analysis and not for construction of test articles. This loss of structural efficiency inherent in an equivalent plate approximation makes the simultaneous matching of the stiffness and frequency difficult. Hence, the use of similarity parameters to scale equivalent plate models should be evaluated to match both stiffness and frequency characteristics of the reference structure.

\section{Concluding Remarks}

A process to generate an equivalent plate based on an optimization approach with a stiffness matching objective function and a frequency matching constraint function was proposed. Several candidate approaches to generating an equivalent plate model of a stiffened wing-like structure were proposed, analyzed, and evaluated. Candidate materials included aluminum, graphite/epoxy, and a graphite/epoxy foam-core sandwich. Candidate modeling approaches included polynomial variation of thickness across the domain, zonal/piecewise constant variation of thickness and density across the domain, and the replacement of the plate mass matrix with one representing point nodal masses.

The reference plate stiffnesses, as represented by its static response to a specified load and boundary condition set, were successfully matched for all of the candidate equivalent plates. The first five natural frequencies of the optimized equivalent plates corresponded very closely to those of the reference plates. The single zone composite

plate produced results were significantly poorer than the other approaches, which all matched frequencies within $3 \%$.

Mode shape fidelity was not a requirement for the equivalent plates, but their mode shapes were examined. In general these mode shapes corresponded well with those of the reference plates. Lower modes matched better than higher modes.

\section{References}

1. Giles, G. L. "Equivalent Plate Modeling for Conceptual Design of Aircraft Wing Structures,” AIAA-19953945, Presented at $1^{\text {st }}$ AIAA Aircraft Engineering, Technology and Operations Congress, Los Angeles, CA, Sept. 19-21, 1995.

2. Mason, B. H., Stroud, W., Krishnamurthy, T., Spain, C., and Naser, A., "Probabilistic Design of a Wind Tunnel Model to Match the Response of a Full-Scale Aircraft,” AIAA-2005-2185, Presented at 46th AIAA/ASME/ASCE/AHS/ASC Structures, Structural Dynamics and Materials Conference 13th AIAA/ASME/AHS Adaptive Structures Conference, Austin, Texas, Apr. 18-21, 2005.

3. Giles, G. L. "Equivalent Plate Analysis of Aircraft Wing Box Structures with General Planform Geometry,” Journal of Aircraft, Vol. 23. No. 11, pp. 858-864, 1986. 
4. Giles, G. L. "Further Generalization of Equivalent Plate Representation for Aircraft Structural Analysis," Journal of Aircraft, Vol. 26. No. 1, pp. 67-74, 1989.

5. Stone, S. C., Henderson, J. L., Nazari, M. M., Boyd, W. N., Becker, B. T., Bhatia, K. G., Giles, G. L. and . Wrenn, G. A., "Evaluation of Equivalent Plate Solution (ELAPS) in HSST Sizing," AIAA-2000-1452, Presented at 41st AIAA/ASME/ASCE/AHS/ASC Structures, Structural Dynamics, and Materials Conference and Exhibit, 41st, Atlanta, GA, Apr. 3-6, 2000.

6. Mavris, D. N., and Hayden, W. T., "Probabilistic Analysis of an HSCT Modeled with an Equivalent Laminated Plate Wing,” AIAA-1997-5571, Presented at AIAA and SAE, 1997 World Aviation Congress, Anaheim, CA, Oct. 13-16, 1997.

7. Krishnamurthy, T. and Mason, B. H., "Equivalent Plate Analysis of Aircraft Wing with Discrete Source Damage”, AIAA-2006-2218, Presented at 47th AIAA/ASME/ASCE/AHS/ASC Structures, Structural Dynamics, and Materials Conference, 14th AIAA/ASME/AHS Adaptive Structures Conference, Newport, Rhode Island, May 1-4, 2006

8. Anonymous, Getting Started with ABAQUS, Version 6.5, ABAQUS, Inc., Providence, RI 02909.

9. Anonymous, DOT, Design Optimization Tools, User's Manual, Version 5.0, Vanderplaats Research \& Development, Inc., Colorado Springs, CO, 80906. 